\title{
Efficacy and specificity of different methods for human neutrophil extracellular trap isolation and handling
}

\author{
FEI GAO $O^{1}, Y U N G U O^{2}$ \\ ${ }^{1}$ Department of Intensive Care Unit, The Affiliated Wuxi People's Hospital of Nanjing Medical University, China \\ ${ }^{2}$ Department of Respiratory Medicine, The Affiliated Wuxi Children's Hospital of Nanjing Medical University, China
}

\begin{abstract}
Introduction: Although in vitro incubation of various cell types with neutrophil extracellular traps (NETs) is commonly used to investigate the influence of NETs on cellular function, it is unclear which human NET isolation and handling protocol is superior. The present study sought to assess the efficacy (yield and purity) and efficiency (time taken) of different available human NET isolation and handling protocols.

Material and methods: Neutrophils isolated from human blood were stimulated using phorbol 12-myristate 13-acetate. Four distinct protocols were used to isolate NETs, and the yield was quantified using fluorimetry.

Results: Addition of the restriction enzyme AluI prior to centrifugation is unique to the most effective NET isolation method, yielding a NET concentration of $1077.22 \pm 229.04 \mathrm{ng} / \mathrm{ml}$ (at $523 \mathrm{~nm}$ ) measured with PicoGreen. Immediate centrifugation to pellet neutrophils is unique to the most efficient method.

Conclusions: Balancing protocol efficacy and efficiency, the method incorporating centrifugation for 5 min at $450 \times \mathrm{g}$ to pellet neutrophils is more than adequate.
\end{abstract}

Key words: neutrophil, neutrophil extracellular traps, isolation, method comparison.

(Cent Eur J Immumol 2021; 46 (3): 384-387)

\section{Introduction}

Neutrophil extracellular traps (NETs) consist of chromatin filaments coated with histones, proteases, and other granular and cytosolic proteins [1]. Recognized as an important neutrophil feature, NET release contributes to microbial capture, immobilization, and killing, but overzealous NET release can significantly damage host tissue [2-4]. Previous studies have demonstrated the substantial contribution of NETs to mortality across multiple disorders, including infectious diseases, autoimmune diseases, cancer, and thrombosis $[4,5]$. However, the mechanisms underlying deleterious NET effects are not yet fully understood.

Although in vitro incubation of cells with NETs is commonly used to evaluate the impact of NETs on cellular function, it is unclear which human NET isolation and handling protocol is superior for this application [6-11]. Therefore, we assessed the efficacy and efficiency of different available protocols.

\section{Material and methods}

\section{Ethical approval and participant consent}

This study was approved by the Ethics Committee of Wuxi Children's Hospital (WXHC2020-03-003). Six children were enrolled as healthy volunteers, and informed consent was provided by the parents of the six children.

\section{Protocol selection and modification}

Four previously described NET isolation and handling protocols were selected and labeled A-D (Table 1) [7-9, $12,13]$. The original protocols specify distinct neutrophil culture conditions and NETosis-activating stimuli, but since the specific goal of the present study was to evaluate NET isolation performance, all other differences were minimized: a single neutrophil isolation method was used, culture conditions were identical, and only phorbol 12-myristate 13-acetate (PMA) (FMS, China, catalog \# FMS-FZ207) was used to stimulate NETosis.

\section{Peripheral blood neutrophil isolation}

Neutrophils were isolated from freshly collected blood samples using a Neutrophil Isolation Kit (TBD, Tianjing, China, catalog \# LZS11131) according to the manufacturer's instructions. Briefly, fresh blood from healthy donors was overlaid onto the neutrophil isolation reagent. After centrifugation for $25 \mathrm{~min}$ at $750 \times \mathrm{g}$, the neutrophilenriched supernatant was collected. Two rounds of residual erythrocyte lysis were followed by centrifugation for

Correspondence: Yun Guo, Department of Respiratory Medicine, The Affiliated Wuxi Children's Hospital of Nanjing Medical University, Qingyang Road, Wuxi, Jiangsu Province, People's Republic of China, 214000, e-mail: guoyun@njmu.edu.cn Submitted: 27.11.2020; Accepted: 23.02.2021 
Table 1. Protocols for NET isolation and handling

\begin{tabular}{|c|c|c|}
\hline Protocol & NET isolation procedure & Reference(s) \\
\hline A & $\begin{array}{l}\text { 1. Gently aspirate and discard culture supernatant. } \\
\text { 2. Using } 15 \mathrm{ml} \text { of cold PBS (lacking } \mathrm{Ca}^{2+} \text { and } \mathrm{Mg}^{2+} \text { ) wash each sample - wash the bottom of each dish } \\
\text { by pipetting PBS on the bottom of the dish to detach all adherent material }- \text { into a } 15 \mathrm{ml} \text { conical tube. } \\
\text { 3. Centrifuge for } 10 \mathrm{~min} \text { at } 450 \times \mathrm{g} \text { and } 4^{\circ} \mathrm{C} \text { to pellet neutrophils, leaving NETs suspended in the supernatant. } \\
\text { 4. Divide the supernatant in each tube into } 1.5 \mathrm{ml} \text { micro-centrifuge tubes and centrifuge for } 10 \text { min } \\
\text { at } 18,000 \times \mathrm{g} \text { and } 4^{\circ} \mathrm{C} \text { to pellet NETs. } \\
\text { 5. Discard supernatants, recombining all pellets deriving from a common } 15 \mathrm{ml} \text { conical tube with } 5 \text { ice cold RPMI. }\end{array}$ & [12] \\
\hline $\mathrm{B}$ & $\begin{array}{l}\text { 1. Gently aspirate and discard culture supernatant. } \\
\text { 2. Using } 5 \mathrm{ml} \text { of fresh RPMI, wash each sample - wash the bottom of each dish by pipetting medium on the } \\
\text { bottom of the dish to detach all adherent material }- \text { into a } 15 \mathrm{ml} \text { conical tube. } \\
\text { 3. Centrifuge for } 5 \mathrm{~min} \text { at } 1500 \mathrm{rpm} / 450 \times \mathrm{g} \text { to pellet neutrophils, leaving NETs suspended in the supernatant. }\end{array}$ & [7] \\
\hline $\mathrm{C}$ & $\begin{array}{l}\text { 1. Gently aspirate and discard culture supernatant. } \\
\text { 2. Add } 5 \mathrm{ml} \text { of fresh RPMI containing } 10 \mathrm{U} / \mathrm{ml} \text { restriction enzyme AluI and incubate neutrophils for } 30 \mathrm{~min} \\
\text { at } 37^{\circ} \mathrm{C} \text {. } \\
\text { 3. Wash the bottom of each dish by pipetting medium on the bottom of the dish and collect each supernatant into } \\
\text { a } 15 \mathrm{ml} \text { conical tube. } \\
\text { 4. Centrifuge for } 5 \mathrm{~min} \text { at } 300 \times \mathrm{g} \text { to pellet any remaining neutrophils, leaving NET fragments suspended } \\
\text { in the supernatant. }\end{array}$ & {$[8,13]$} \\
\hline $\mathrm{D}$ & $\begin{array}{l}\text { 1. Wash the bottom of each dish by pipetting medium on the bottom of the dish, collect all neutrophils } \\
\text { and supernatants into a } 15 \mathrm{ml} \text { conical tube. } \\
\text { 2. Centrifuge for } 5 \mathrm{~min} \text { at } 500 \times \mathrm{g} \text { to pellet neutrophils. } \\
\text { 3. Transfer supernatants to fresh } 1.5 \mathrm{ml} \text { micro-centrifuge tubes. } \\
\text { 4. Centrifuge for } 10 \mathrm{~min} \text { at } 18,000 \times \mathrm{g} \text { and } 4^{\circ} \mathrm{C} \text { to pellet NETs. } \\
\text { 5. Discard supernatants, recombining all pellets deriving from a common } 15 \mathrm{ml} \text { conical tube with } 5 \text { fresh RPMI. }\end{array}$ & [9] \\
\hline
\end{tabular}

$10 \mathrm{~min}$ at $450 \times \mathrm{g}$. Neutrophils were washed twice with phosphate-buffered saline (PBS) (Bioind, Israel, catalog \# 02-024-1ACS) and resuspended in Roswell Park Memorial Institute (RPMI) 1640 medium (Bioind, Israel, catalog \# 01-100-1ACS) supplemented with 3\% fetal bovine serum (FBS) (Bioind, Israel, catalog \# 04-001-1ACS) $\left(1 \times 10^{6}\right.$ cells $\left./ \mathrm{ml}\right)$.

\section{Neutrophil stimulation and NET isolation}

A volume corresponding to $5 \times 10^{6}$ neutrophils was inoculated into $25 \mathrm{~cm}^{2}$ cell culture flasks. After incubation with $500 \mathrm{nM}$ PMA for $4 \mathrm{~h}$ in a $37^{\circ} \mathrm{C}$ incubator with $5 \% \mathrm{CO}_{2}$, neutrophils were subjected to NET isolation protocols A-D. Each protocol was repeated at least in sextuplicate.

\section{Quantification of NETs}

A PicoGreen dsDNA kit (Thermo Fisher Scientific, Waltham, USA, catalog \# P7589) was used for NET quantitation according to the manufacturer's instructions. Briefly, a total of $100 \mu \mathrm{l}$ of isolated NET was transferred to 96 -well plates, followed by $100 \mu \mathrm{l}$ of $1 \mathrm{X}$ Quant-iT PicoGreen reagent. Plates were incubated for $5 \mathrm{~min}$ at room temperature prior to fluorescence detection (excitation/ emission wavelengths: $502 / 523 \mathrm{~nm}$ ) using a SpectraMax M2 spectrofluorometer (Molecular Devices, Biberach an der Riß, Germany).

\section{Statistical analysis}

All statistical analyses were performed using SPSS Statistics 23.0 for Windows 10 (IBM, New York, U.S.). A one-way analysis of variance (ANOVA) was used to analyze the differences between groups. For multiple comparisons, Dunnett's test was selected when one column represented control data, and the Tukey test was selected when comparing all pairs of columns. All values representing normally distributed data are expressed as the mean \pm standard deviation. A $p$-value $<0.05$ was considered statistically significant.

\section{Results}

Although protocol C (Addition of the restriction enzyme AluI prior to centrifugation) is most effective, yielding a NET concentration of $1077.22 \pm 229.04 \mathrm{ng} / \mathrm{ml}$, protocol B (Immediate centrifugation to pellet neutrophils) is the most efficient, with implementation times of only 15 min each (Table 2). All protocols yielded highly pure NET fractions free of neutrophil contamination (Fig. 1).

\section{Discussion and conclusions}

To the best of our knowledge, this is the first study comparing the efficacy and efficiency of different human NET isolation and handling protocols. Protocol C was 
Table 2. Yield and purity resulting from protocols for neutrophil extracellular trap (NET) isolation and handling

\begin{tabular}{lccl}
\hline Protocol & $\begin{array}{c}\text { Duration } \\
(\mathbf{m i n})\end{array}$ & $\begin{array}{c}\text { DNA concentration } \\
\text { measured } \\
\text { by PicoGreen }(\mathbf{n g} / \mathbf{m l})\end{array}$ & $\begin{array}{c}\text { Cellular } \\
\text { contamination }\end{array}$ \\
\hline $\mathrm{A}$ & 30 & $244.13 \pm 150.96$ & Absent \\
\hline $\mathrm{B}$ & 15 & $657.17 \pm 105.47$ & Absent \\
\hline $\mathrm{C}$ & 40 & $1077.22 \pm 229.04$ & Absent \\
\hline $\mathrm{D}$ & 20 & $299.86 \pm 106.13$ & Absent \\
\hline
\end{tabular}

Data are presented as mean $\pm S D$
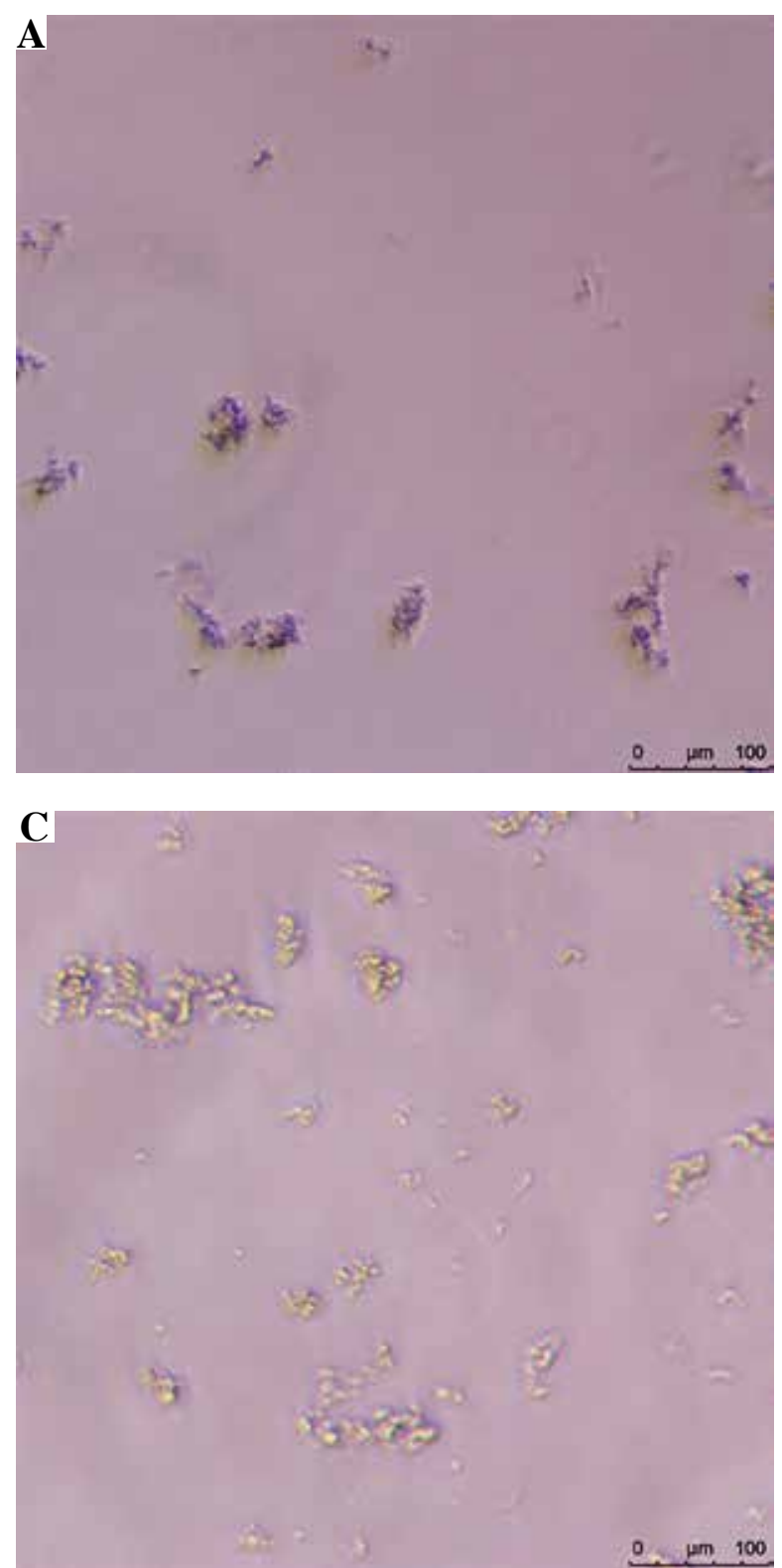

found to be most effective, generating the highest NET yield. This may be attributable to the inclusion of the restriction enzyme AluI, which cleaves DNA at 5'-AG/CT-3' recognition sites to generate small NET fragments [14], thereby minimizing loss during centrifugation. In addition, because DNA fragmentation will significantly affect DNA concentration as estimated using fluorimetry, this method may significantly underestimate yields resulting from protocol C [15]. Although protocols A and D both generated highly pure NET fractions, their yields were relatively low, likely due to inclusion in these protocols of double the number of centrifugation steps relative to other protocols.
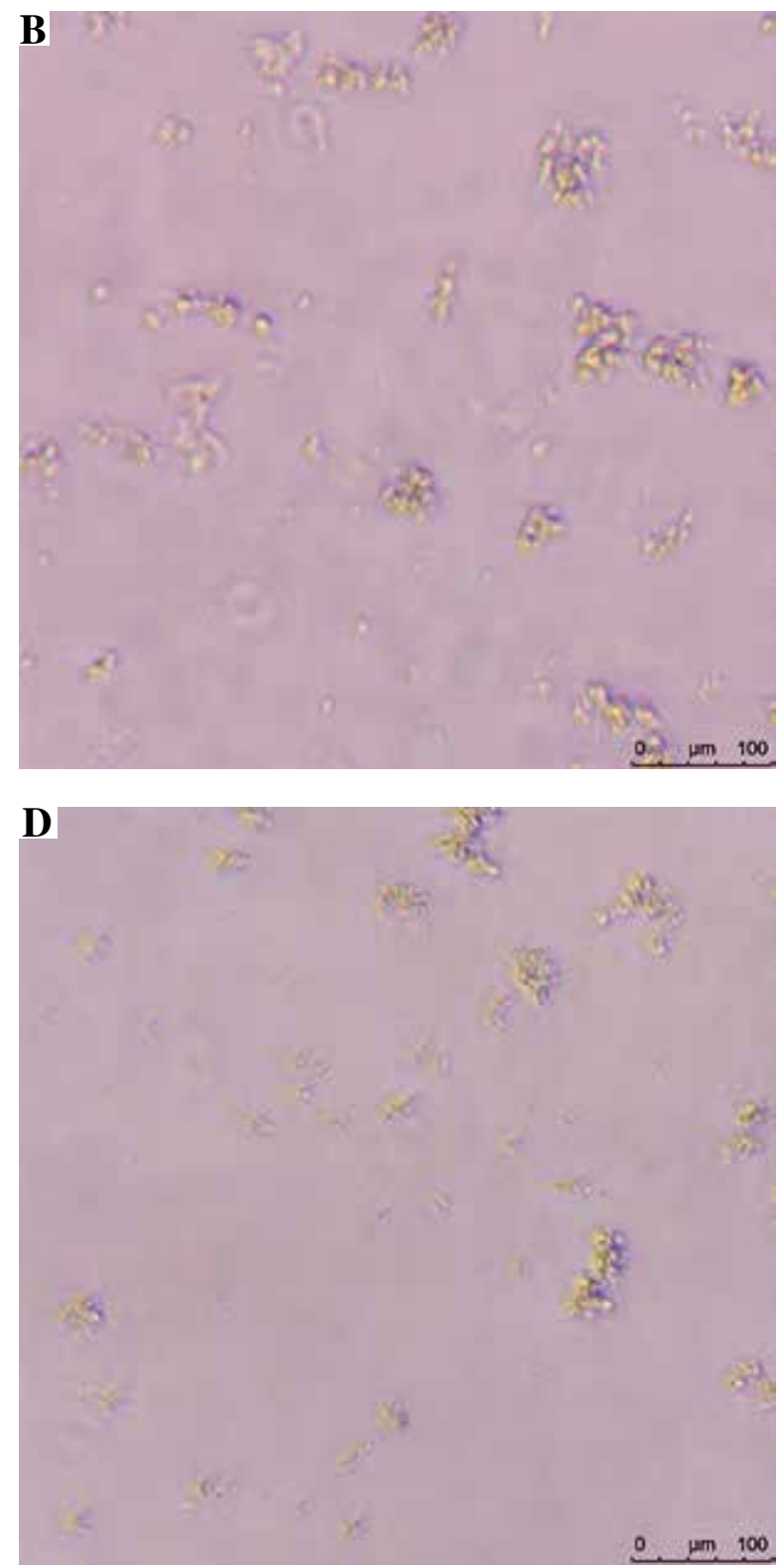

Fig. 1. Photomicrographs of purified NETs (20× magnification). Scale bar: $100 \mu \mathrm{m}$ 
In addition, both protocols $\mathrm{A}$ and $\mathrm{D}$ require access to an ultracentrifuge. Finally, it is worth noting that if pathogens replace PMA as NETosis triggers, they may prove difficult to remove via subsequent centrifugation during protocol $\mathrm{D}$.

In conclusion, although protocol $\mathrm{C}$ produced the highest NET yield, balancing protocol efficacy and efficiency suggests that protocol B (incorporating centrifugation for $5 \mathrm{~min}$ at $450 \times \mathrm{g}$ to pellet neutrophils) is more than adequate.

\section{Funding}

This study was funded by the Young Project of Wuxi Health and Family Planning Commission (grant number Q202054), the Major Program of Wuxi Health and Family Planning Commission (grant number Z202016), and the Wuxi Maternal and Child Health Research Project (grant number FYKY202003).

\section{Ethical approval and participant consent}

This study was approved by the Ethics Committee of Wuxi Children's Hospital (WXHC2020-03-003). Six healthy children were enrolled in this study and informed consent was obtained from the parents of all individual participants.

The authors declare no conflict of interest.

\section{References}

1. Burgener SS, Schroder K (2020): Neutrophil extracellular traps in host defense. Cold Spring Harb Perspect Biol 12: a037028.

2. Twaddell SH, Baines KJ, Grainge C, Gibson PG (2019): The emerging role of neutrophil extracellular traps in respiratory disease. Chest 156: 774-782.

3. Castanheira F, Kubes P (2019): Neutrophils and NETS in modulating acute and chronic inflammation. Blood 133: 2178-2185.

4. Hughes MJ, Sapey E, Stockley R (2019): Neutrophil phenotypes in chronic lung disease. Expert Rev Respir Med 13: 951-967.

5. Soehnlein O, Steffens S, Hidalgo A, Weber C (2017): Neutrophils as protagonists and targets in chronic inflammation. Nat Rev Immunol 17: 248-261.

6. Golbach LA, Scheer MH, Cuppen JJ, et al. (2015): Low-frequency electromagnetic field exposure enhances extracellular trap formation by human neutrophils through the NADPH pathway. J Innate Immun 7: 459-465.

7. Farrera C, Fadeel B (2013): Macrophage clearance of neutrophil extracellular traps is a silent process. J Immunol 191: 2647-2656.

8. Mangold A, Hofbauer TM, Ondracek AS, et al. (2019): Neutrophil extracellular traps and monocyte subsets at the culprit lesion site of myocardial infarction patients. Sci Rep 9: 16304.

9. Barr FD, Ochsenbauer C, Wira CR, Rodriguez-Garcia M (2018): Neutrophil extracellular traps prevent HIV infection in the female genital tract. Mucosal Immunol 11: 1420-1428.
10. Saffarzadeh M, Juenemann C, Queisser MA, et al. (2012): Neutrophil extracellular traps directly induce epithelial and endothelial cell death: a predominant role of histones. PLoS One 7: e32366.

11. Frangou E, Chrysanthopoulou A, Mitsios A, et al. (2019): REDD1/autophagy pathway promotes thromboinflammation and fibrosis in human systemic lupus erythematosus (SLE) through NETs decorated with tissue factor (TF) and interleukin-17A (IL-17A). Ann Rheum Dis 78: 238-248.

12. Najmeh S, Cools-Lartigue J, Giannias B, et al. (2015): Simplified human neutrophil extracellular traps (NETs) isolation and handling. J Vis Exp 98: 52687.

13. Silvestre-Roig C, Braster Q, Wichapong K, et al. (2019): Externalized histone $\mathrm{H} 4$ orchestrates chronic inflammation by inducing lytic cell death. Nature 569: 236-240.

14. Smith DR (1993): Restriction endonuclease digestion of DNA. Methods Mol Biol 18: 427-431.

15. Sedlackova T, Repiska G, Celec P, et al. (2013): Fragmentation of DNA affects the accuracy of the DNA quantitation by the commonly used methods. Biol Proced Online 15: 5 . 